Revue internationale P.M.E.

Économie et gestion de la petite et moyenne entreprise

Revue

internationale

PME

\title{
Pour une approche renouvelée des structures organisationnelles des ME-MI françaises
}

\section{Yvan Leray}

Volume 12, numéro 4, 1999

URI : https://id.erudit.org/iderudit/1008664ar

DOI : https://doi.org/10.7202/1008664ar

Aller au sommaire du numéro

Éditeur(s)

Presses de l’Université du Québec

ISSN

0776-5436 (imprimé)

1918-9699 (numérique)

Découvrir la revue

Citer cet article

Leray, Y. (1999). Pour une approche renouvelée des structures organisationnelles des ME-MI françaises. Revue internationale P.M.E., 12(4), 33-55. https://doi.org/10.7202/1008664ar
Résumé de l'article

À l'appui d'une étude qualitative portant sur les pratiques structurelles de 24 moyennes entreprises et industries françaises, cet article s'interroge sur la prégnance de la forme fonctionnelle.

Après avoir évoqué des raisons d'ordre épistémologique et théorique, pratique et culturel, cet article propose une approche renouvelée tenant compte de nouveaux paramètres de structuration. 


\title{
Pour une approche renouvelée des structures organisationnelles des ME-MI françaises
}

Yvan LERAY

Université de Rennes I

\author{
MOTS CLÉS
}

Moyennes entreprises et industries - France

Structure organisationnelle - Analyse qualitative - Configurations

Paramètres de structuration

\begin{abstract}
RÉSUMÉ
À l'appui d'une étude qualitative portant sur les pratiques structurelles de 24 moyennes entreprises et industries françaises, cet article s'interroge sur la prégnance de la forme fonctionnelle.

Après avoir évoqué des raisons d'ordre épistémologique et théorique, pratique et culturel, cet article propose une approche renouvelée tenant compte de nouveaux paramètres de structuration.
\end{abstract}

\section{ABSTRACT}

Based on a qualitative study of 24 French medium-sized firms and their structural systems, this article estimates the prevalence of the functional form.

After examining of epistemological and theoretical, practical and cultural reasons, this article propounds a renewed approach with new structurating parameters.

\section{L'AUTEUR}

YVAN LERAY est titulaire d'un doctorat en sciences de gestion de l'Université de Rennes I, jeune chercheur au CREREG et membre associé au CNME (IAE Caen). II occupe aussi la fonction d'ATER à la Faculté de droit et des sciences économiques du Mans. Ses recherches portent sur le management et l'organisation des moyennes entreprises et industries. Adresse: CREREG, Institut de gestion de Rennes, 11, rue Jean-Macé ; B.P. 1997, 35019 Rennes, Cedex, France. Téléphone: 33299847834 (secrétariat CREREG). Télécopieur : 33299847800. 


\section{RESUMEN}

Basándose en un estudio cualitativo sobre las costumbres/hábitos estructurales de 24 PyMEs e industrias francesas, este artículo evalúa el predominio de la forma funcional.

Después de evocar razones de orden epistemológico y teórico, práctico y cultural, este artículo propone un enfoque renovado que tiene en cuenta nuevos parámetros de estructuración.

\section{ZUSAMMENFASSUNG}

Dieser Artikel hinterfragt die Pragmatik funktionaler Formen in Anlehnung an eine qualitative Studie von 24 französischen mittleren Industriebetrieben.

Nachdem die "epistemologischen", theoretischen, praktischen und kulturellen Hintergründe benannt worden sind, konnte anhand dieses Artikels eine neue Erkenntnis unter Berücksichtigung neuer struktureller Parameter festgestellt werden.

L'idée que l'organisation de taille moyenne s'affirme comme le référent organisationnel non seulement pour la grande entreprise mais aussi par rapport à la vie économique française ${ }^{1}$ constitue certainement une première explication de la multiplication des investigations sur le champ spécifique mais contingent (Torrès, 1997) des moyennes entreprises et industries ${ }^{2}$. Si on s'interroge sur leurs atouts (Gélinier, 1996 ; Joffre et Wickham, 1997), leurs structures de propriété (Le Vigoureux, 1997), leurs pratiques stratégiques (Saporta, 1997), technologiques (Julien et Carrière, 1994) ou humaines (Bayad, 1995 ; Mahé de Boislandelle, 1998), par exemple, les travaux portant sur leurs aspects organisationnels sont moins fréquents.

Bien qu'il existe quelques interrogations et travaux sur le sujet (Echène, 1973 ; Basire, 1976 ; Gervais, 1978 ; Kalika, 1988), force est de constater qu'ils datent au mieux d'une décennie. Si ceux-ci restent des référentiels, les évolutions

1. Entre 1985 et 1996 , la variation des effectifs salariés est de $+12,5 \%$ pour les $\mathrm{ME}$ françaises (50-199 salariés), celle des MGE (200-499) est de $+0,03 \%$ alors que celle des GE (500 et +) est de $-23,9 \%$, enfin, celle des PE (10-49) est de $+14,8 \%$; d'après la synthèse de Boyer et Germain, 1998.

2. Il est entendu par ME-MI toute entité économique et sociale identifiée, instrument d'un projet, qui satisfait à la production de biens ou de services. Parce qu'il n'est actuellement pas possible de se référer à un critère de classification des PME unique et partagé (Julien, 1990 ; Duchéneaut, 1995), le critère de reconnaissance retenu est la taille en termes d'effectifs salariés, conformément à la récente nomenclature de l'Union européenne, « les moyennes entreprises comprennent de 50 à 199 salariés » (Marchesnay et Fourcade, 1997), et à la classification du GREPME - Revue internationale PME. 
concurrentielles (mondialisation, orientation, marché) et technologiques (systèmes d'informations intégrés, nouvelles technologies), d'une part, et les nouveaux modes opératoires de gestion (JAT, flux tendus) et d'organisation du travail (travail cellulaire, compétences), d'autre part, invitent à s'interroger sur les formes organisationnelles adoptées par les ME-MI.

En prenant pour paramètres de structuration (internes) le degré de centralisation et le nombre de niveaux hiérarchiques, deux tendances configurationnelles ${ }^{3}$ sont couramment admises ${ }^{4}$. Ou bien la forme organisationnelle est entrepreneuriale, c'est-à-dire construite autour du dirigeant. Ou bien la forme organisationnelle est fonctionnelle, c'est-à-dire construite selon les grandes fonctions nécessaires à la réalisation de son activité stratégique (forme proche de la tendance bureaucratique). S'il est vrai que cette approche a le mérite d'offrir une vision synthétique, elle n'évite toutefois pas l'écueil de la simplification excessive.

En effet, si la forme entrepreneuriale est un référent incontournable, particulièrement lorsque la ME-MI a un effectif avoisinant les 50 salariés, une monoactivité et un dirigeant-propriétaire, le fait que la forme fonctionnelle à tendance bureaucratique constitue l'autre référent est plus discutable. Dans un contexte où la transversalité organisationnelle (processus transversal de satisfaction du client, circulation latérale de l'information, gestion de projets) l'emporte sur le cloisonnement fonctionnel, on peut s'étonner de la prégnance de la forme fonctionnelle dans l'approche des ME-MI.

De fait, on perçoit l'intérêt de mettre à jour la connaissance des structures des moyennes organisations en répondant à deux questions. La prégnance de la forme fonctionnelle est-elle justifiée ? Ne peut-on pas envisager une approche plus fine des formes organisationnelles adoptées par les ME-MI?

\section{La prégnance de la forme fonctionnelle est-elle justifiée?}

Afin de répondre à cette question, nous utilisons les résultats d'une étude qualitative des pratiques de définition structurelle de $24 \mathrm{ME}-\mathrm{MI}$ françaises. Nous verrons qu'il existe bien souvent un décalage entre un «énoncé formel » fonctionnel et la réalité de la structure organisationnelle. Sur la base de ces résultats, nous essaierons d'expliquer la prégnance fonctionnelle dans les moyennes organisations françaises.

3. Une configuration est une combinaison multidimensionnelle d'éléments qui apparaissent concomitamment à un temps donné. Une approche configurationnelle permet de postuler que la multiplicité structurelle peut être limitée à quelques tendances regroupant de manière cohérente un certain nombre de caractères.

4. Voir Duchéneaut, 1996, p. 225. 


\subsection{Invitation au dépassement de la forme fonctionnelle: les résultats d'une étude des pratiques de définition structurelle}

L'étude dont nous présentons ici les principaux résultats repose sur l'observation de pratiques de définition structurelle d'entreprises et d'industries de taille moyenne, singulièrement de celles ayant adopté une forme fonctionnelle. Chaque observation a pris appui sur une interview centrée, composée d'une série de questions cherchant à mesurer d'éventuels décalages entre la structure formelle définie dans chaque entreprise et des critères internes de structuration (voir une synthèse de la méthode, encadré 1).

Cette étude à caractère exploratoire (Leray, 1998) montre que parmi les entreprises à forme fonctionnelle rencontrées, la moitié admettent un décalage entre «l'énoncé formel» et des critères internes de structuration.

\section{ENCADRÉ 1 \\ Cadre méthodique de l'étude des pratiques de définition structurelle de ME-MI}

L'objet de l'étude était d'analyser les pratiques de définitions structurelles de ME-MI françaises. Sa mise en place s'est appuyée sur l'utilisation d'une typologie ${ }^{1}$ de profils configurationnels ${ }^{2}$ tirée de la littérature (cf. annexe II). Ces simplifications compréhensives (chaque profil) de la réalité organisationnelle des ME-MI ont été utilisées comme des guides à l'observation de leurs pratiques structurelles. En utilisant ce cadre logique comme point de repère, nous avons développé une étude des arrangements organisationnels de 24 ME-MI de secteurs d'activité différents. La méthode d'observation et d'analyse de ces arrangements reposait sur trois étapes :

- une radiographie de chaque organisation rencontrée à travers un entretien (cf. annexe I) de type focused interview ${ }^{3}$ construit autour de deux thématiques : i) identitaire, afin de positionner l'organisation (âge, taille, secteur d'activité, rôle de la structure, stratégie de l'entreprise, environnement économique et social, âge du dirigeant, ancienneté du dirigeant dans la fonction, formation du dirigeant, etc.) ; ii) comportementale, afin de saisir la pratique de définition structurelle (type d'organigramme, nombre de niveaux hiérarchiques, niveau de délégation des tâches de direction, type de coordination, etc.);

- une lecture comparative de chaque organisation prenant pour point de départ des variables de structuration internes (style de direction, préoccupation du dirigeant, type de coordination) rattachées de manière univoque aux cinq profils configurationnels (boîte à outils conceptuelle ${ }^{4}$, Véran, 1995) précédemment évoqués et permettant de constater, pour chaque organisation, d'éventuels 
Bien que l'existence de décalages entre le formel et le pratiqué ne soit pas réellement surprenant, car les structures ne sont que des représentations du mode d'action, utiles comme facteur d'intégration des acteurs de l'entreprise (Kalika, 1997) mais ponctuelles, il ressort une similitude dans les décalages mis au jour. Il apparaît, pour les ME-MI rencontrées, un décalage entre la représentation du fonctionnement organisationnel (définition structurelle) et les moyens structurels mis en œuvre pour atteindre un objectif stratégique de rapprochement du marché. Par exemple, pour cette moyenne industrie de génie électrique et travaux publics (130 salariés, chiffre d'affaires de 75 millions de francs) structurée sous forme fonctionnelle, le choix des variables structurelles (style de direction plus participatif que délégatif ; préoccupation de la direction plus tournée vers le marché et la consolidation de l'organisation que vers la productivité ; coordination plus par les résultats que par la délégation) fait apparaître un décalage. Si l'on ajoute à ces éléments que, pour le dirigeant, la structure organisationnelle doit permettre la mobilisation (ici, impact d'une démarche qualité indissociable de l'implication des acteurs), la

décalages entre la structure telle qu'elle est définie et les caractères pris par ces trois variables. Il est à noter que l'objectif de l'étude n'était pas de rechercher des décalages à tout prix. Parmi les entités rencontrées ayant une définition fonctionnelle de leur structure, la moitié admettait un décalage ${ }^{5}$;

- une mise en lumière d'enseignements de manière à dégager toute l'expérience du terrain d'investigation, c'est-à-dire de manière à marquer des similitudes dans les pratiques de définition structurelles de ME-MI.

La présentation de cette étude, ainsi que l'exposition des premiers résultats, a fait l'objet d'une communication aux XIVes Journées nationales des IAE en juin 1998 à Nantes. Afin de lever toute ambiguiité et erreur d'interprétation, nous tenons à préciser que cette étude n'a aucune valeur statistique et n'avait pas pour objectif de vérifier l'existence des profils configurationnels par des tests quantitatifs. Il s'agissait ici d'observer la réalité de pratiques de définition structurelle à travers le prisme d'une méthode partant du typologique.

1. Définition wébérienne d'une typlogie : accentuation unilatérale d'un ou plusieurs caractères et enchaînement d'un ensemble de phénomènes isolés afin de former un tableau de pensée homogène, voir Freund, 1968.

2. Définition d'une configuration : combinaison multidimensionnelle d'éléments qui apparaissent concomitamment à un temps donné, voir le Forum dans l'Academy of Management Journal, vol. 36, nº 6, décembre 1993.

3. Voir Morin, 1984.

4. Voir Van de Ven, 1989.

5. Trois structures simples, une structure préfonctionnelle, 14 structures fonctionnelles, six structures quasi divisionnelles, aucune structure situationnelle. 
communication et l'adaptabilité (ici, remarquable dans le recours à une flexibilité des moyens humains), on confirme le dépassement de «l'énoncé formel » fonctionnel.

Dans la mesure où l'identification de décalages n'est pas synonyme de remise en cause de la pérennité de l'entreprise, ils prennent une valeur relative. En revanche, si l'on n'y prête pas attention, on entretient une contre-vérité. Accepter les décalages, c'est admettre un reniement des théories de la contingence (Burns et Stalker, Woodward, Lawrence et Lorsch), pour partie issues d'observations de moyennes organisations. En d'autres termes, et même s'il convient d'avoir une vision nuancée de la contingence aujourd'hui (Leray, 1999c), il n'empêche qu'une théorie qui avance qu'à un environnement complexe correspond une structure organique et qu'à un environnement simple correspond une structure mécanique reste valable. On peut alors être étonné que certaines ME-MI choisissent une forme fonctionnelle à tendance bureaucratique pour favoriser leur adaptation au marché. À partir du moment où un décalage apparaît, le choix des variables structurelles montre qu'une forme analytique paraît mieux adaptée à un changement de paradigme organisationnel : triptyque qualité-coût-délai ; nouvelles formes d'organisation du travail (unité élémentaire de travail, équipe cellulaire, logique de projet) et de la production (Kanban, juste-à-temps) facilitées par les nouvelles technologies de l'information ; stratégie orientée client (service, innovation, rapidité).

Par ailleurs, on peut être étonné que certains travaux avancent l'idée que les moyennes entreprises seraient des bureaucraties décentralisées (Dubost, 1995). Bien que ces travaux soient statistiquement validés, ils restent largement hérités d'études sur les configurations de grande taille. La spécificité organisationnelle des ME-MI (moindre formalisation et standardisation, mélange des fonctions) montre que l'on ne peut se satisfaire du réductionnisme de la grande entreprise. Il nous semble aujourd'hui nécessaire de compléter la perception habituellement partagée sur les organisations de taille moyenne, mais qui date d'une décennie déjà ${ }^{5}$. Il apparaît tout aussi nécessaire de dépasser la «vision mythique des PME » (Marchesnay, 1991) inspirée des travaux de Mintzberg mais qui reste cultivée dans les faits puisque l'on continue trivialement à prendre pour base de réflexion le type entrepreneurial ou le type bureaucratie professionnelle.

À ce sujet, une étude menée par Dolty, Glick et Huber (1993) montre que l'approche configurationnelle de Miles et Snow (1978) est plus pertinente que celle de Mintzberg $(1982,1990)$. En procédant à des tests comparatifs, cette étude permet une plus grande représentativité de l'approche en quatre configurations de Miles

5. C'est l'habituelle référence à la recherche de Kalika (1988) dont $75 \%$ des entreprises de l'échantillon (79 entreprises) était composé d'entités de 50 à 200 salariés ayant majoritairement adopté une forme fonctionnelle (80\% des 50-200 salariés), pour seulement $12,5 \%$ pour la forme entrepreneuriale et $7,5 \%$ pour la forme par produit. 
et Snow avec un accent mis sur la configuration analyser proche du type quasi divisionnel dans la mesure où elle est un mélange de la configuration defender (fonctionnelle) et de la configuration prospector (divisionnelle), la quatrième configuration étant la configuration reactor (situationnelle). Cette étude invite donc à voir les configurations pour la moyenne taille autrement qu'à travers le prisme de la forme fonctionnelle (tendance bureaucratique), la forme entrepreneuriale étant, quant à elle, plus «naturelle».

Avec cet ensemble d'éléments, nous pouvons affimer que la prégnance fonctionnelle n'est pas justifiée. Il peut en effet exister des décalages entre des «énoncés formels » fonctionnels et les paramètres de définition structurelle plutôt divisionnels. Dès lors, on est amené à se demander pourquoi la forme fonctionnelle reste le référent théorique, avec la forme simple (entrepreneuriale), des structures des organisations de taille moyenne. Un essai d'explication de cette prégnance fonctionnelle pour les ME-MI françaises peut être proposé.

\subsection{Essai d'explication de la prégnance fonctionnelle}

Afin de comprendre la prégnance fonctionnelle, un ensemble de raisons d'ordre épistémologique et théorique, pratique et culturel peut être évoqué :

- D'un point de vue épistémologique, le fait même de parler de fonction (Kalika, 1978) conduit à envisager les structures sous l'angle de regroupements d'autorité selon des fonctions verticales spécialisées (les six F de Fayol). C'est là une première explication de la prégnance fonctionnelle. Une deuxième raison tient dans trois héritages : i) l'héritage d'une conception fonctionnaliste des économistes selon laquelle l'entreprise («boîte noire ») est un agent de production combinant des facteurs de production pour transformer des input en output; ii) 1'héritage d'un structurofonctionnalisme des sociologues (Parsons, 1961) qui propose comme impératifs fonctionnels de tout système social l'atteinte d'un but et le maintien de la stabilité ; iii) l'héritage, peut-être insuffisamment contesté, du « paradigme fonctionnaliste » des gestionnaires qui invite à considérer les structures organisationnelles en se polarisant sur la division taylorienne du travail, la spécialisation des tâches, le mécanisme de coordination, et qui, plus largement, ne tient compte que de la définition structurelle. On voit bien dans l'étude (cf. supra) que, même en envisageant l'approche des structures à travers leur définition et des éléments internes, mais en considérant ces éléments de manière concomitante, on dépasse cet héritage fonctionnaliste. Nous croyons dès lors que le poids du paradigme fonctionnaliste relève plus d'une certaine forme de complaisance des théoriciens (c'est la question de l'innovation méthodologique et du dépassement de l'approche configurationnelle qui est posée) et des praticiens 
(c'est de la sauvegarde des pouvoirs du dirigeant dont il est question) envers une forme qui, nous le verrons, offre un cadre de protection au dirigeant.

On peut aussi évoquer le poids des référents théoriques invitant à percevoir le comportement organisationnel des ME-MI comme bureaucratique. Par exemple, en conclusion de son étude, Kalika avance que dans «les structures de taille moyenne, les caractéristiques " bureaucratiques " que sont la standardisation, la formalisation et la centralisation sont synonymes d'une plus grande efficacité de l'entreprise » $\left(\right.$ Kalika, 1988) ${ }^{6}$. Si ce résultat constitue une base de connaissance essentielle, il nous semble qu'il tient en grande partie à l'instrumentation statistique de l'étude. Ainsi, notre travail ne tend à avancer que lorsqu' on aborde la question des structures d'entreprises et d'industries moyennes avec une méthode d'investigation centrée sur leur dynamique organisationnelle, on complète foncièrement cette base de connaissance.

Toujours d'un point de vue théorique, il nous paraît insuffisant de corréler une supposée tendance des PME à la bureaucratie à l'augmentation d'un contrôle externe source de perte d'indépendance financière (ouverture du capital social, intégration dans un groupe) ou économique (poids des donneurs d'ordres, concentration des débouchés). Si ces éléments sont importants, il n'est pas certain qu'ils les brident dans l'adoption de formes moins mécaniques et plus orientées vers le marché. Par exemple, la mise en réseau permet d'éviter l'obstacle de la faiblesse des ressources.

Hier comme aujourd'hui (Basire, 19767 ; Greeman, 1994), la moyenne organisation, notamment la moyenne entreprise industrielle, reste définie comme fonctionnelle avec une primauté de la production et de l'administration, puis dans une seconde mesure de la commercialisation (et de la R-D). Une explication plausible réside dans les limites cognitives du dirigeant (notamment entrepreneur) qui, pour des besoins d'encadrement, fait appel à un spécialiste, et parfois à plusieurs (directeurs de production, commercial, financier) fonctionnels. Une autre explication relève de l'idée que la structure doit être garante de rationalité.

Une dernière raison théorique tient dans la perception, à notre avis discutable, d'une moyenne organisation comme phase transitoire à la grande organisation. Ainsi, dans une logique de développement planifié des structures (évolution graduelle), l'étape de la moyenne taille est celle devant permettre l'affirmation

6. M. Kalika (1988), Structures d'entreprises, Paris, Economica, p. 413 ; malgré des relations structure-efficacité relativement lâches (p. 414).

7. Basire propose une moyenne entreprise industrielle scindée en deux classes : a) une classe 50-70 salariés avec l'affirmation de la fonction productive mais aussi de la fonction administrative ; b) une classe 71-200 salariés avec l'autonomie de la fonction commerciale. 
fonctionnelle. Une augmentation de la taille rime avec une hausse de la fonctionnalisation. Or, différents éléments nous ont donné l'occasion de remettre en cause cette idée. Les interprétations configurationnelles présentées ci-avant laissent entrevoir les moyennes organisations sous un autre angle que le fonctionnel. En outre, si la ME-MI n'était que le reflet d'une transition, comment expliquer que la grande organisation, mais aussi la moyenne grande organisation et la moyenne organisation, fassent des choix organisationnels d'éclatement de structure, notamment dans un souci de mieux «coller» au marché ou de «capter» des opportunités locales d'implantation ? C'est l'illustration donnée par cette moyenne industrie indépendante spécialisée dans la fabrication d'ovoproduits (70 salariés, chiffre d'affaires de 172 millions de francs dont $60 \%$ à l'export) qui opte pour une croissance par filialisation. Si on ne peut nier l'existence d'une certaine redondance des problèmes internes (limites cognitives du dirigeant, manque de compétence et formation collective, dépendance parfois trop grande à l'égard des conseils extérieurs, consolidation des actifs, problème de la transmission), on ne peut accepter sans restriction l'idée que les ME-MI, et plus largement les PME-PMI, souffriraient «d'une maladie de langueur» (Girard, 1998). Le fait d'envisager différentes déclinaisons structurelles pour les ME-MI, tout en gardant à l'esprit leur hétérogénéité, est peut-être une voie pour se dégager de la prégnance fonctionnelle.

- D'un point de vue pratique, il est clair que le dirigeant façonne directement ou indirectement la structure organisationnelle, que ce soit en tenant compte de son comportement, de son système de valeurs (Bamberger, 1982) ${ }^{8}$, de son «need for achievement » (Miller et Dröge, 1986), de son profil sociologique (Lewin et Stephens, 1994). Dès lors, la structure fonctionnelle sert parfois de refuge de rationalité pour les dirigeants face aux changements stratégiques (buts, activité, marché), mais aussi psychosociologiques (anxiété, relations interpersonnelles) et politiques (jeux de pouvoir internes) que l'on sait être des composants majeurs de l'interprétation des phénomènes structurels (Desreumaux, 1992).

Une autre explication pratique tient dans le manque de considération pour la consolidation de l'organisation. Même s'il n'est pas question de proposer «la » structure performante, on sait en revanche qu'un mauvais choix structurel nuit à l'efficacité des entreprises. Par ailleurs, dire que pour les organisations de taille moyenne il s'agit essentiellement de s'adapter à l'instabilité environnementale nous paraît incomplet. Leur adaptation à l'environnement passe aussi par la maîtrise de leur propre rythme et de leur capacité de changement permanent. Des efforts d'adaptation mal cadencés avec le fonctionnement structurel restent fréquemment sans effet. Aussi, on peut avancer que la nécessaire adaptation permanente à l'environnement n'apparaît réalisable que si l'entreprise mène une réflexion préalable sur

8. Les réflexions de Bamberger portaient sur les PME. 
la manière dont elle est structurée. La seule réactivité (répondre aux stimuli extérieurs) est insuffisante (Godet, 1991). Le fait de mener une réflexion sur l'organisationnel peut aider, notamment lorsqu'elle est combinée à une réflexion sur la compétence (Leray et Joyeau, 1998), à mieux préparer le changement (préactivité). Aussi, il semble plus réaliste de considérer à la fois l'influence extérieure (par exemple, la compréhension de l'évolution des besoins des clients) et la capacité d'agencement organisationnel (par exemple, la définition d'une organisation selon le processus de satisfaction du client).

On peut avancer un dépassement de l'unique référence au modèle fonctionnel et afficher un intérêt particulier pour une structure plus analytique, plus divisionnelle. Si le choix d'une forme fonctionnelle semble adapté à une entreprise de taille moyenne recherchant principalement la productivité, rien ne dit qu'il en soit de même dans un contexte d'adaptabilité où l'orientation produit-marché-client est préférée. L'expérience des ME-MI rencontrées montre que lorsqu'il est question de diversification de l'activité stratégique, de diversité des attentes du client, de développement à l'export, d'innovation (produit, procédé, organisation), la tendance divisionnelle semble la plus adaptée.

Le dernier type d'explications a trait à une dimension culturelle. On peut tout d'abord évoquer la perception collective d'une ME-MI ayant une mono-activité, un fonctionnement organisationnel hiérarchisé (centralisation de la décision) et contrôlé, une forme organisationnelle rationnelle et fonctionnelle. Or, dans un contexte de pression concurrentielle, une diversification d'activités est possible. C'est le cas de cette entreprise industrielle de 130 salariés ayant deux activités (le génie électrique et les TP) et une organisation quasi divisionnelle liée aux chantiers. Or, dans un contexte de mondialisation, on ne peut ignorer que des ME-MI, parfois leader sur leur marché (Simon, 1998), travaillent à l'export (cas d'une entreprise de fabrication de spiritueux de 183 salariés) en adoptant une organisation par clients. Or, dans un contexte de banalisation des produits, l'innovation passe par une gestion transversale de projets qui bouscule les frontières internes et externes de l'entreprise.

Comparativement aux États-Unis et au Japon, si la forme fonctionnelle est prévalante en France (et plus largement en Europe), bien qu'elle soit évaluée comme la moins efficiente ${ }^{9}$ (Clark et Fujimoto, 1991), notamment en raison d'une moindre circulation de l'information (Aoki, 1988), cela tient aussi aux propriétés socioculturelles françaises telles que l'individualisme, une crainte à l'égard de l'incertitude, un certain éloignement relationnel entre strates hiérarchiques (Bollinger et Hofstede, 1987), ainsi qu'à une sorte de fatalisme franco-français à croire en un environnement démiurge contre lequel il est nécessaire de se protéger ${ }^{10}$ en privilégiant une

9. «Modèle séquentiel » moins efficient que le « modèle du dialogue » privilégiant le projet.

10. Culture d'entreprise défensive, Burke (1987). 
permanence organisationnelle définie comme la seule réponse appropriée. Ces facteurs induisent une tendance à la centralisation (jacobinisme et dirigisme), à la formalisation (besoin de sécurité et cartésianisme), à la spécialisation (d'un côté, les ingénieurs et de l'autre, les commerciaux) et à l'élitisme ${ }^{11}$. Les caractéristiques de la forme fonctionnelle (centralisation, standardisation, cloisonnement) constituent alors des mécanismes de défense contre le changement, des résistances au processus d'apprentissage au sens d'Argyris (1998).

\section{Pour une approche renouvelée des formes organisationnelles en ME-MI}

Au-delà de l'essai d'explication de la prégnance fonctionnelle pour les ME-MI françaises, on pressent l'intérêt de renouveler la connaissance de leurs formes organisationnelles par une autre voie qu'un réductionnisme de la grande taille ou qu'une vision statistique de leur réalité. Tout en tenant compte de la diversité des situations possibles et en conservant un point de départ interne à l'approche des structures organisationnelles des ME-MI, il peut s'agir d'opter pour une approche qui s'intéresse à leur fonctionnement structurel.

C'est dans cette optique que nous pouvons faire état de pratiques structurelles de ME-MI. Prenant pour point d'appui une méthode originale (Leray, 1999a) combinant une approche configurationnelle et une approche interactionniste (voir encadré 2), quatre études de cas approfondies ont été menées. Dans l'ensemble, les enseignements issus des quatre études de cas in situ conduisent à proposer deux types de résultats confirmant un dépassement de la prégnance fonctionnelle : un changement de nature du soutien fonctionnel et la considération de nouveaux paramètres de structuration.

\subsection{Changement de nature du soutien fonctionnel comme confirmation du dépassement de la prégnance fonctionnelle en ME-MI}

En s'intéressant au fonctionnement quotidien d'organisations de taille moyenne, nous avons constaté différentes similitudes. En prenant pour point de départ l'interne, il semble que les variables de standardisation, de formalisation et de planification-contrôle revêtent une importance moindre dans la structuration des ME-MI. La décision et la coordination prennent une dimension synthétique pour qualifier leurs formes organisationnelles (cf. annexe I).

11. Burke (1987), p. 48-50, dirigisme, cartésianisme, élitisme. 


\section{ENCADRÉ 2 \\ Cadre méthodique de l'étude du fonctionnement structurel de ME-MI}

L'objet de l'étude était d'analyser le fonctionnement structurel de ME-MI françaises. En s'appuyant sur quatre études de cas in situ, cette étude avait pour finalité de mieux comprendre les pratiques structurelles actuelles d'organisations de taille moyenne. Il s'agissait ici de s'intéresser au comportement structurel sous l'angle des interactions entre acteurs salariés, c'est-à-dire d'envisager la dynamique organisationnelle en considérant les flux opérationnels et de travail comme fondateurs du fonctionnement structurel.

Pour ce faire, et parce que l'approche interactionniste véhicule une méthode particulière, le développement et l'utilisation d'un outil composé d'un programme informatique (utilisation compilée de différents outils informatiques : Visual Basic, Lotus Approach, Excel) ont permis d'automatiser l'analyse des structures des moyennes organisations. Reposant sur l'analyse structurale de réseau, une des branches des mathématiques, cet outil présente l'avantage de mettre en avant des processus d'interaction réguliers liés au fonctionnement opérationnels de ME-MI. Révélateur de comportement structurel, l'outil est apparu comme une manière d'objectiver les réalités organisationnelles rencontrées et, plus particulièrement, comme une manière de mesurer la transversalité organisationnelle. Il s'agit là de la quatrième avancée de la recherche.

Selon les buts à atteindre et l'évolution environnementale, les ME-MI privilégient aussi des éléments actuels tels que le processus de satisfaction du client, les équipes de processus, les flux d'informations, la transversalité organisationnelle, la compétence des individus.

Si ces éléments obligent à renouveler l'approche des structures des ME-MI (voir ci-après le point 2.2.), ils induisent également un changement de nature du soutien fonctionnel, autant au niveau du soutien technique qu' au niveau du soutien logistique.

En ce qui concerne le soutien technique (c'est-à-dire des « analystes » de la technostructure), on constate qu'il concerne moins la standardisation du travail des membres de l'organisation (logique procédurière) que l'aide technique et d'expertise à la réalisation des processus. Par exemple, dans une entreprise indépendante de conception, fabrication et commercialisation de robinetterie industrielle (67 salariés, chiffre d'affaires 36260000 F) il s'agit moins, dans une logique de JAT et de standardisation modulable de la gamme de produits, de planifier la production que d'envisager une adaptation permanente de l'ordonnancement de la production aux variations des commandes. 
Plus que la dimension mathématique de l'outil, son développement montre que l'on peut contribuer à la connaissance des structures des ME-MI françaises (Leray, 1999a) autrement qu'à travers une approche configurationnelle avançant une vision statique de l'organisation. Généralement tirée du seul point de vue de ses dirigeants et donnant un poids important à la définition structurelle, au formel, cette approche évite difficilement deux écueils : celui qui, en s'appuyant sur une démarche uniquement statistique, conduit à produire une vision de la moyenne taille déduite de ce qui se passe pour la grande taille (écueil du réductionnisme) et celui qui conduit à n'envisager la question organisationnelle des ME-MI qu'en référence à des travaux globaux ignorant leurs spécificités (écueil de la vision mythique).

En revanche, lorsque l'approche configurationnelle permet de produire des simplifications compréhensives (ou profils configurationnels) de la réalité tirées de réflexions intégrant la taille moyenne et suffisamment nuancées pour servir de boîte conceptuelle (et non pas de cadre modélisé), on est en mesure d'apporter une connaissance critique (cf. l'examen de la prégnance fonctionnelle) des arrangements organisationnels de la ME-MI. Lorsque cette approche se trouve combinée à une approche interactionniste cherchant à comprendre le comportement et la dynamique organisationnelle de la ME-MI (voir supra), on est en mesure d'apporter une connaissance renouvelée (cf. l'approche renouvelée, 2.2.) du fonctionnement organisationnel de la ME-MI. C'est dans cette combinaison des conceptions configurationnelle et interactionniste dans ce qu'elles ont de pertinent par rapport à une mise à jour de la connaissance théorique et pratique des structures des moyennes organisations françaises (1996-1998) que réside toute l'originalité d'une recherche doctorale dont nous présentons ici les grands enseignements.

S'agissant du soutien logistique (c'est-à-dire qui a vocation à rendre des services internes tels que la restauration, l'entretien, etc.), lorsqu'il existe, on remarque qu'il tient plus un rôle de fourniture de moyens matériels et humains que d'apport de services indépendants du processus de réponse au client. Par exemple, dans l'entreprise de travaux publics et de génie électrique déjà citée, le service des moyens intervient directement dans la réalisation des chantiers autant pour des aspects matériels et humains que pour des aspects de conseil et de contrôle de la qualité. En règle générale, il semble que le soutien logistique est très peu développé au sein des ME-MI en raison d'un effet de taille évident. Lorsqu'il existe, il est intégré à d'autres composants organisationnels (pour l'entreprise de robinetterie industrielle, la tarification est assurée par le commercial, les relations sociales directement assurées par le P.-D. G. ou le D. G.) ou bien tend à être externalisé.

Ces deux modifications concourent à moduler le modèle mintzberguien de dynamique organisationnelle et à modérer l'utilisation sans restriction du référent bureaucratique (forme fonctionnelle). À cet égard, le fait que les référents théoriques et pratiques antérieurs à cette étude datent un peu est sans nul doute un élément à prendre en considération, notamment en raison d'une affirmation de nouveaux 
modes de management. Par exemple, la qualité en exerçant tout à la fois un contrôle (conformité du produit ou service, et de la tâche), une standardisation (maîtrise des procédures) et du conseil (mise en place de la démarche qualité et amélioration continue) est plus une fonction de support transversal, orientée et intervenant directement à plusieurs étapes du processus de réponse au client, qu'une fonction de support vertical uniquement orientée vers la standardisation.

Dans l'ensemble, il apparaît que le changement de nature du soutien fonctionnel est corrélé à la capacité d'anticipation des besoins du client et à la possibilité de standardisation des réponses données au client. On remarque, en effet, deux cas de figure : i) l'affirmation d'un soutien fonctionnel (technique ou logistique) lorsque la ME-MI doit apporter une variété de réponses au client ; ii) moins de soutien fonctionnel lorsqu'il existe une unité des réponses. Dans une entreprise indépendante franchisée de restauration rapide (68 salariés), face à une attente connue, voire des flux de fréquentation anticipés, le soutien fonctionnel est très faible (sauf quand il s'agit du lancement d'un nouveau produit, ce qui passe par un effet standardisation des tâches en amont du lancement). Dans un établissement bancaire, face à une logique de produits à la carte, la nécessité d'un soutien fonctionnel se fait sentir. Ce qui prime ici, c'est le contact avec le client. Par exemple, la spécificité du problème d'héritage d'un portefeuille boursier à la suite d'un décès oblige à faire appel à une expertise juridique. La particularité de la création d'entreprise pose des problèmes de maîtrise de viabilité, de solvabilité, de levée des risques que la personne en contact avec le client ne mesurera pas forcément. Notons que rien n'interdit de penser que, dans un avenir proche, les NTIC et la puissance des systèmes d'informations permettront une déconcentration du soutien directement au niveau du terrain (idée de «banque éclatée » ${ }^{12}$ ).

\subsection{Prise en compte de nouveaux paramètres de structuration des ME-MI}

L'étude de la dynamique organisationnelle de quelques moyennes entreprises et industries nous conduit à mettre l'accent (voir le schéma infra) sur de nouveaux paramètres de structuration. Selon les buts à atteindre et l'évolution environnementale, la nouvelle approche repose sur trois dimensions :

- une dimension organisationnelle avec, d'un côté, une tendance à l'horizontalité (mouvance structurelle, décentralisation de la décision, activité des membres coordonnée par ajustement) pour des équipes de processus de satisfaction du client variables et un poids important des flux d'informations, et, de l'autre, une tendance à la verticalité (permanence structurelle,

12. L. Bryan (1989), Banque éclatée, Paris, InterÉditions. 
centralisation de la décision, activité des membres coordonnée par supervision) pour des équipes de processus de satisfaction du client standard et un poids important des flux physiques ;

- une dimension client avec la variété ou l'unité du processus de satisfaction $d u$ client (une commande dans les entreprises de robinetterie et de restauration, un chantier dans l'entreprise de TP et d'installations électriques, un service dans l'établissement bancaire) et la prise en compte de la capacité (plus ou moins développée) de standardisation des réponses à apporter, voire d'anticipation de ses besoins ;

- une dimension humaine avec la relation de l'homme à son travail avec, d'un côté, l'emploi et la compétence, et, de l'autre, la tâche et la qualification.

Si l'on ajoute à ces dimensions la possibilité de positionner différents équilibres comportementaux, ou simplifications compréhensives de la réalité (voir les cinq configurations, annexe II), on offre une vision actuelle des structures organisationnelles des ME-MI. Aussi, bien que cette schématisation relève moins de la modélisation que de la contribution à la connaissance, elle nous paraît constituer un référentiel qui non seulement affirme un dépassement de la prégnance fonctionnelle mais qui confirme également une rupture avec le réductionnisme de la grande taille vers la petite taille.

FIGURE 1

Configurations et nouveaux paramètres de structuration de ME-MI

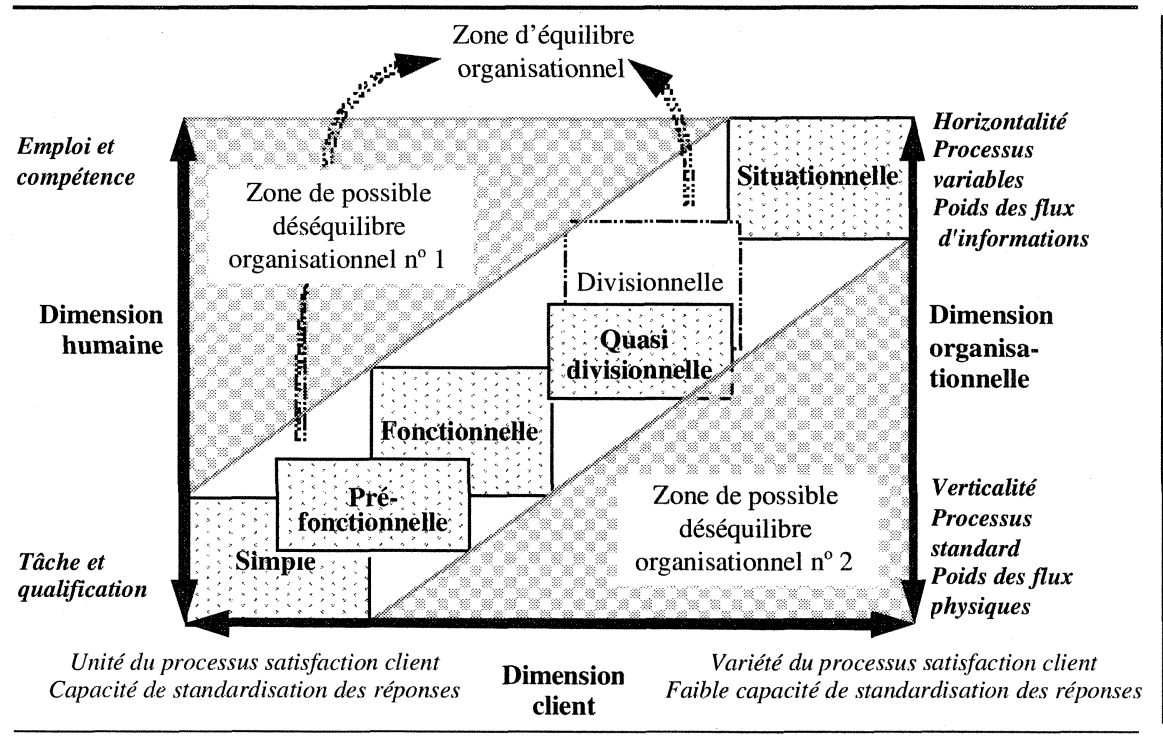


L'apport de cette schématisation paraît double. Tout d'abord, elle souligne l'intérêt d'identifier et de répondre aux besoins du client et pas seulement de pousser une gamme de produits ou services sur le marché, a fortiori dans les ME-MI où les orientations stratégiques prises engagent leur survie. L'approche de leur dimension structurelle doit tenir compte de cette donnée. C'est pourquoi il faut aussi prendre en considération la capacité d'anticipation des besoins du client (dimension client et stratégie), l'importance des flux d'informations ou physiques et l'existence d'équipes de processus variables ou standard (dimension organisation, système de production, technologie et organisation du travail), le niveau de formation des acteurs, leur autonomie, leur mobilité, leur maîtrise des nouvelles technologies (dimension humaine et culture d'entreprise). On a ainsi la possibilité de produire une vision proche de la réalité des ME-MI.

Ensuite, elle peut aider au positionnement de la gestion organisationnelle. Selon la logique économique dans laquelle se trouve une ME-MI (logique de l'offre avec une poussée des produits ou services sur le marché versus logique de la demande avec une recherche de la satisfaction du client), on envisagera, par exemple, les cas suivants :

- la réalisation de l'activité relève de la tâche pour un ou des processus de satisfaction du client standard avec une relation de l'homme à son travail définie par le poste et la qualification, on tend alors vers une affirmation de la verticalité hiérarchique (cas de l'entreprise de restauration rapide) ou fonctionnelle (cas d'une entreprise mono-activité sous dépendance économique d'un donneur d'ordres);

- la réalisation de l'activité relève de la compétence pour un ou des processus de satisfaction du client variables avec une relation de l'homme à son travail définie par l'emploi et la connaissance, on tend alors vers une affirmation de l'horizontalité divisionnelle (cas des entreprises de robinetterie industrielle, de génie électrique et TP, et bancaire).

Dans le même ordre d'idées, en proposant des zones de déséquilibre organisationnel possible, elle peut aider à la réflexion sur la gestion organisationnelle, voire sur la conduite du changement. On comprend, par exemple:

- qu'une ME-MI ayant la possibilité de standardiser la réponse à apporter au client n'a pas intérêt à mettre en œuvre un mode d'action horizontal faisant appel à l'emploi et à la compétence de l'ensemble de ses salariés (zone de possible déséquilibre $n^{0} 1$, figure 1 ), même si une démarche qualité peut introduire une transversalité organisationnelle, sauf si l'entreprise cherche à créer une rupture favorable à l'innovation (produit, procédé, organisation); 
- qu'une ME-MI soumise à une variété de la demande de ses clients peut difficilement la satisfaire par un mode d'action vertical faisant appel à la tâche et à la qualification de ses salariés (zone de possible déséquilibre $n^{0} 2$, figure 1), même si on ne peut ignorer qu'il subsiste des logiques organisationnelles bureaucratiques dans certaines entités du service public, sauf à considérer que la bureaucratie est l'idéal type organisationnel (Weber) ou à renoncer aux fondements de l'École de la contingence, ce qui est loin d'être notre cas.

\section{Conclusion}

Cet article montre que les formes entrepreneuriale et fonctionnelle ne sauraient à elles seules qualifier un univers de gestion des ME-MI spécifique et hétérogène. Même si la réalité des ME-MI n'est jamais univoque dans le sens où les possibilités d'agencements sont multiples, il peut être intéressant de proposer des représentations permettant d'accroître leur connaissance. Dans cette optique, nous avons pu proposer différentes simplifications compréhensives (ou configurations) des modalités organisationnelles des ME-MI. Reflets d'équilibres comportementaux, elles permettent d'éclairer et d'expliquer la réalité des structures organisationnelles des ME-MI, et d'envisager leur évolution ${ }^{13}$.

Dans cet article, nous avons pu voir qu'à travers une étude qualitative des pratiques de définition structurelle de $24 \mathrm{ME}-\mathrm{MI}$ françaises la prégnance de la forme fonctionnelle n'est pas justifiée mais qu'elle peut être expliquée par différentes raisons d'ordre épistémologique et théorique, pratique et culturel. En s'attardant sur le fonctionnement organisationnel de certaines d'entre elles, nous avons remarqué un changement de nature du soutien fonctionnel comme confirmation du dépassement de la prégnance fonctionnelle en ME-MI, d'une part, et nous avons envisagé une schématisation combinant de nouveaux paramètres de structuration avec différentes configurations de base comme guide à la compréhension de l'organisation de la ME-MI, d'autre part.

Parce qu'elle peut aider le chercheur à « organiser son savoir » sur l'organisation des ME-MI, parce qu'elle peut aider le gestionnaire en «lui évitant de se perdre dans les détails et en lui favorisant le regroupement de données empiriques éparses ${ }^{14}$ », parce qu'elle suscite de nouvelles voies de recherche, nous pensons que nous sommes peut-être en présence d'un modèle utile, à tout moment modifiable cependant par de nouveaux résultats ou des approches différentes.

13. «A good theory explains, predicts, and delights (Weick, 1995).

14. N. Aubert et al. (1996), Management. Aspects humains et organisationnels, Paris, Presses universitaires de France, p. 29. 


\section{ANNEXE I}

\section{Structure de l'interview centrée}

IDENTIFICATION

Organisation :

- Dénomination de l'organisation.

- Secteur d'activité.

- Nom du dirigeant.

- Effectifs actuels (1996) \effectifs passés (1992-1995).

- Type d'organisation (entreprise personnelle et familiale, autre entreprise indépendante, filiale, établissement) et statut juridique.

- Âge de l'organisation.

- Chiffre d'affaires, évolution, part à l'export.

- Mesure d'efficacité (organisationnelle, sociale, économique), voir Kalika, 1988, p. 333.

\section{Dirigeant :}

- Dirigeant (âge, sexe, statut, ancienneté dans la fonction, situation antérieure au poste de direction, niveau de formation, formation de base, moyen de perfectionnement).

- Prendre une décision, c'est : chiffrer, trouver une solution logique à des problèmes et des faits ; agir, réaliser au quotidien, répondre à des problèmes les uns à la suite des autres ; utiliser et accumuler de l'expérience, faire référence à l'intuition, à la sensibilité ; anticiper, se projeter dans l'avenir, favoriser l'innovation et les idées.

- Stratégie du dirigeant (croissance, rentabilité).

- La satisfaction de votre stratégie passe par l'action de : renforcer l'activité sur un marché acquis ; rechercher de nouveaux débouchés pour des produits existants ; augmenter les ventes grâce à de nouveaux produits sur le marché connu ; rechercher de nouveaux clients en leur proposant de nouveaux produits).

\section{COMPORTEMENT}

- Type de structure et informations sur l'organigramme (historique, répartition des rôles, nombre de niveaux hiérarchiques, degré de subordination, date de formulation, etc.).

- Degré de délégation de la tâche de direction.

- Style de direction, préoccupation de la direction.

- Type de coordination.

- Type de souci organisationnel auquel doit répondre la structure (adaptabilité, flexibilité, productivité, communication, coordination, contrôle, décision, innovation, relation, etc.). 


\section{Tableau des différents profils configurationnels (Leray, 1999 a, b)}

\begin{tabular}{|c|c|c|c|c|c|}
\hline \multirow[b]{2}{*}{$\begin{array}{l}\text { Dimensions structurelles } \\
\text { et contextuelles }\end{array}$} & \multirow{2}{*}{$\begin{array}{l}\text { Tendance hiérarchique } \\
\text { Structure simple }\end{array}$} & \multicolumn{2}{|c|}{ Tendance fonctionnelle } & \multirow{2}{*}{$\begin{array}{l}\text { Tendance analytique } \\
\text { Structure quasi divisionnelle }\end{array}$} & \multirow{2}{*}{$\begin{array}{l}\text { Tendance réactive } \\
\text { Structure situationnelle }\end{array}$} \\
\hline & & Structure préfonctionnelle ${ }^{1}$ & Structure fonctionnelle & & \\
\hline Style de direction & Entrepreneurial et individualiste & Directif & Délégatif & Participatif & Collégial \\
\hline $\begin{array}{l}\text { Centralisation/ } \\
\text { Décentralisation }\end{array}$ & Centralisation & $\begin{array}{l}\text { Décentralisation verticale } \\
\text { partielle (délégation de } \\
\text { certaines tâches) }\end{array}$ & $\begin{array}{l}\text { Décentralisation verticale } \\
\text { (décision opérationnelle) }\end{array}$ & $\begin{array}{l}\text { Décentralisation horizontale et } \\
\text { verticale (décision } \\
\text { opérationnelle tactique) }\end{array}$ & $\begin{array}{l}\text { Décentralisation (décision } \\
\text { opérationnelle, tactique et en } \\
\text { partie stratégique) }\end{array}$ \\
\hline Coordination interne & $\begin{array}{l}\text { Supervision directe (régulation } \\
\text { par le dirigeant) }\end{array}$ & $\begin{array}{l}\text { Supervision (régulation par } \\
\text { voie hiérarchique) }\end{array}$ & Responsabilisation & Résultats & Ajustement réciproque \\
\hline Spécialisation liée à & $\begin{array}{l}\text { (plutôt) une polyvalence des } \\
\text { membres de l'oganisation }\end{array}$ & des tâches (début) & $\begin{array}{l}\text { des tâches, des qualifications } \\
\text { pour les responsables de } \\
\text { fonctions }\end{array}$ & $\begin{array}{l}\text { des qualifications (ou des } \\
\text { objectifs }^{2} \text { ) }\end{array}$ & des savoirs et compétences \\
\hline $\begin{array}{l}\text { Préoccupation de la } \\
\text { direction }^{3}\end{array}$ & Fabrication & Fabrication / Vente & Productivité & $\begin{array}{l}\text { Marché et consolidation de } \\
\text { l'organisation }\end{array}$ & Innovation \\
\hline \multicolumn{6}{|l|}{ Contextuelles } \\
\hline Système technique & $\begin{array}{l}\text { Système technique simple lié à } \\
\text { une production à l'unité - à la } \\
\text { demande (petite série) }\end{array}$ & $\begin{array}{l}\text { Système technique } \\
\text { préautomatisé lié à une } \\
\text { production de masse } \\
\text { (moyenne série) }\end{array}$ & $\begin{array}{l}\text { Système technique automatisé } \\
\text { lié à une production de masse } \\
\text { (grande série) } \\
\text { technologie routinière }\end{array}$ & $\begin{array}{l}\text { Système technique très } \\
\text { automatisé lié à une } \\
\text { production - processus }\end{array}$ & $\begin{array}{l}\text { Système hautement } \\
\text { technologique totalement lié } \\
\text { à l'information }\end{array}$ \\
\hline Environnement & Simple et dynamique & Simple et stable & Complexe et stable & Complexe et instable & Complexe et très instable \\
\hline Pouvoir & Autoritaire (voire paternaliste) & Autoritaire & Technocratique & $\begin{array}{l}\text { Décentralisé et rapproché } \\
\text { du marché }\end{array}$ & Démocratique? \\
\hline Design & $\begin{array}{l}\text { Plutôt haut avec une } \\
\text { prédominance du sommet } \\
\text { hiérarchique (forme } \\
\text { pyramidale) }\end{array}$ & $\begin{array}{l}\text { Assez proche de la structure } \\
\text { simple avec un aplatissement de } \\
\text { la pyramide (forme pyramidale } \\
\text { avec un élargissement à partir } \\
\text { du milieu) }\end{array}$ & $\begin{array}{l}\text { Aplatie } \\
\text { (forme de râteau) }\end{array}$ & $\begin{array}{l}\text { «Épaissie » autour } \\
\text { des fonctions } \\
\text { ou des produits, } \\
\text { ou des projets (?) }\end{array}$ & $\begin{array}{l}\text { Libre et à variabilité } \\
\text { permanente } \\
\text { (forme proche des idées de } \\
\text { trèfle, de grappe, de réseau, etc.) }\end{array}$ \\
\hline
\end{tabular}

1. On fait ici référence à une forme de structure proposée par Kalika,1988.

2. On pense ici à la structure quasi divisionnelle à base produit.

3. Les préoccupations ici présentées ne doivent pas être perçues comme se substituant à la préoccupation principale de toute organisation: la pérennité / la finalité. 


\section{Bibliographie}

AOKI, M. (1988), Information, Incentives, and Bargaining Structure in Japanese Economy, Cambridge, Cambridge University Press, 320 p.

ARGYRIS, C. (1998), « Le prochain défi », dans F. Hesselbein, M. Goldsmith et R. Beckhard (dir.), L'Entreprise de demain/La Fondation Drucker, Paris, Village Mondial, p. 222-230.

BAmberger, I. (1982), «Les valeurs des dirigeants de PME et leur influence sur le comportement stratégique et la performance de leurs entreprises », Économies et Sociétés, SG no 3, p. 1353-1383.

BASIRE, M. (1976), «La théorie des cinq niveaux », Direction et Gestion des Entreprises, $\mathrm{n}^{\circ} 2$, p. 11-21; no 3, p. 11-22; no 4, p. 13-20; n 5 , p. 11-16; n ${ }^{\circ} 6$, p. $18-34$.

BAYAD, M. (coord.) (1995), «La GRH dans les PME», Gestion 2000, vol 11, n 1, janvierfévrier, p. 91-202.

Bollinger, D. et G. Hofstede (1987), Les différences culturelles dans le management, Paris, Les Éditions d'Organisation, 268 p.

Boudon, R. (1968), À quoi sert la notion de structure?, Paris, Gallimard.

Boyer, L. et O. Germain (1998), «Les entreprises en France : taille et emploi », Cahiers de recherche IAE Caen, $\mathrm{n}^{\circ} 13$.

BRECHET, J.-P. (1997), «Les structures : leur importance, leur inertie, leur flexibilité », Direction et Gestion des Entreprises, n 106, juin-août 1997, p. 17-25.

BURKE, M. (1987), À chacun son style d'entreprise, Paris, InterÉditions, 205 p.

Clark, K.B. et T. Fujimoto (1991), Product Development Performance, Boston, Harvard Business School Press, 409 p.

DESCHAMPS, J.-P. et P.R. NAYAK (1997), Les maîtres de l'innovation totale, Paris, Les Éditions d'Organisation.

Desreumaux, A. (1992), Structures d'entreprise, Paris, Vuibert Gestion.

DESREUMAUX, A. (1996), « Nouvelles formes d'organisation et évolution de l'entreprise », Revue française de gestion, $\mathrm{n}^{\circ} 107$, janvier-février, p. 86-108.

DOLTY, D.H., W.H. GLICK et G.P. HUBER (1993), «Fit, equifinality, and organizational effectiveness : a test of two configurational theories », Academy of Management Journal, vol. 36, nº 6, p. 1196-1250.

Dubost, N. (1995), «Les PME françaises évoluent-elles vers des bureaucraties? », Actes $4^{e}$ Conférence internationale de management stratégique, AISM, vol. 2, mai, p. 596-611.

DuchÉNeAut, B. (1995), Enquête sur les PME françaises, Paris, Maxima.

DuCHÉnEAUT, B. (1996), Les dirigeants de PME, Paris, Maxima.

DURKHEIM, E. (1937), Les règles de la méthode sociologique, 8 édition, 1996, Paris, Presses universitaires de France, Collection «Quadrige».

ECHÈNE, D. (1973), «Le moyenne entreprise, système d'organisation spécifique », Direction et Gestion des Entreprises, $\mathrm{n}^{\circ}$ 6, p. 7-16. 
Freund, J. (1986), Sociologie de Max Weber, Paris, Presses universitaires de France.

GALBRAITH, J.R. (1998), «L'organisation reconfigurable », dans F. Hesselbein, M. Goldsmith et R. Beckhard (dir.), L'Entreprise de demain /La Fondation Drucker, Paris, Village Mondial, p. 86-98.

GASSE, Y. (1991), «L'utilisation de diverses techniques et pratiques de gestion dans la $\mathrm{PME}$ », Revue internationale PME, vol. 4, no 1, p. 3-11.

GÉLINIER, O. (1996), La réussite des entreprises familiales. Les moyennes entreprises patrimoniales, un atout pour l'avenir, Paris, Maxima.

GERVAIS, M. (1978), «Pour une théorie de l'organisation PME», Revue française de gestion, $\mathrm{n}^{\mathrm{o}} 15$, mars, p. 37-49.

GIRARD, B. (1998), «Pourquoi les PME restent-elles PME?», Problèmes économiques, $\mathrm{n}^{\circ}$ 2551, janvier, p. 11-17.

Godet, M. (1991), «Les dangers de la (seule) réactivité », Revue française de gestion, $\mathrm{n}^{\circ} 86$, novembre-décembre.

GREEMAN, N. (1994), «L'organisation du travail dans les PMI se distingue-t-elle de celle des grandes entreprises? », Économie et statistique, $\mathrm{n}^{\circ}$ 271-272, p. 87-103.

JOFFRE, P. et S. WICKHAM (1997), «Les atouts des entreprises moyennes », Revue française de gestion, $\mathrm{n}^{\mathrm{o}} 116$, novembre, p. 64-70.

JULIEN, P.A. (1990), «Vers une typologie multicritère des PME», Revue internationale $P M E$, vol. 3, $\mathrm{n}^{\text {os }} 3-4$, p. 411-425.

Julien, P.A. et J.B. CARrière (1994), «L'efficacité des PME et les nouvelles technologies », Revue d'économie industrielle, $\mathrm{n}^{\circ}$ 67, $1^{\mathrm{er}}$ trimestre, p. 120-134.

KALIKA, M. (1978), Une méthode de diagnostic d'entreprise : la matrice des structures, Thèse de $3^{\mathrm{e}}$ cycle, Université de Poitiers, juillet, p. 42.

KaliKa, M. (1988), Structures d'entreprises, Paris, Economica (2 ${ }^{\mathrm{e}}$ tirage, 1995).

KALIKA, M. (1997), «Organigramme : organisation pratique de l'entreprise », dans Y. Simon et P. Joffre (dir.), Encyclopédie de gestion, tome 2, Paris, Economica, p. $2156-2172$.

LE VigoureuX, M. (1997), «Entreprises moyennes : structures de propriété et comportement stratégique », Revue française de gestion, $\mathrm{n}^{\circ} 116$, novembre, p. 71-84.

LERAY, Y. (1998), «Les structures des moyennes organisations : une étude configurationnelle exploratoire », Actes XIV es Journées nationales des IAE, tome 3, Nantes, avril.

LERAY, Y. (1999a), Structures des moyennes organisations. Contribution à la connaissance des structures des moyennes organisations françaises (1996-1998) par la mise en place d'une approche combinant le configurationnel et l'interactionnisme, Thèse de doctorat Mention «Sciences de Gestion », Université de Rennes I, janvier.

LERAY, Y. (1999b), «Contribution à la connaissance des structures organisationnelles des ME-MI », Direction et gestion, La Revue des sciences de gestion, $\mathrm{n}^{\circ} 168$, novembredécembre 1997 , p. 39-48, et $\mathrm{n}^{\mathrm{o}}$ 175-176, $1^{\text {er }}$ trimestre, p. 71-85. 
LERAY, Y. (1999c), « Théories de la contingence et évolution des structures d'entreprises, une relecture par la Complexité », dans B. Paulré et al. (dir.), Approches évolutionnistes de la firme et de l'industrie, Paris, L'Harmattan, p. 97-114.

LERAY, Y. et A. JOYEAU (1998), «Gestion des compétences et gestion de la structure organisationnelle : l'expérience d'un établissement bancaire de taille moyenne», $2^{e}$ Forum de centre normand de la moyenne entreprise, Caen, IAE-Université de Caen, novembre.

LEWIN, Y. et C.U. STEPHENS (1994), «CEO attitudes as determinants of organizational design : an integrated model », Organization Studies, vol. 15, n 2, p. 183-212.

LOUART, P. (1996), «L'apparente révolution des formes organisationnelles », Revue française de gestion, $\mathrm{n}^{\circ}$ 107, janvier-février, p. 74-85.

MAHÉ DE Boislandelle, H. (1998), «GRH en PME. Universalité et contingences : essai de théorisation », Revue internationale PME, vol. 11, n 2-3, p. 11-30.

MARCHESNAY, M. (1982), «Is small so beautiful ?», Revue d'économie industrielle, $\mathrm{n}^{\circ}$ 19, $1^{\mathrm{er}}$ trimestre.

MARCHESNAY, M. (1991), «Mintzberg on PME», Revue internationale PME, vol. 4, $\mathrm{n}^{\circ}$ 1, p. 131-138.

MArchesnay, M. (1992), «La PME : une gestion spécifique ?», Problèmes économiques, $\mathrm{n}^{\mathrm{o}} 2276$, mai, p. 26-32.

Marchesnay, M. et C. FourCade (dir.) (1997), Gestion de la PME / PMI, Paris, Nathan, Collection «Étapes références ».

Meyer, A.D., A.S. Tsui et C.R. Hinnings (1993), «Configurational approaches to organizational analysis », Academy of Management Journal, vol. 36, nº 6, p. 11751195.

MiLeS, R.E. et C.C. SNOw (1978), Organizational Strategy, Structure and Process, New York, McGraw-Hill, 274 p.

MILLER, D. et C. DRÖGE (1986), «Psychological and traditional determinants of structure», Administrative Science Quarterly, vol. 31, décembre, p. 539-560.

MiLls, C.W. (1967), L'imagination sociologique, Paris, François Maspero, 229 p.

MinTZBERG, H. (1982), Structure et dynamique des organisations, Paris, Les Éditions d'Organisation.

MinTZBERG, H. (1990), Le management. Voyage au centre des organisations, Paris, Les Éditions d'Organisation.

Morin, E. (1984), Sociologie, édition 1994, Paris, Seuil, Collection «Essais », p. 231-249.

PARSONS, T. (1961), «Une esquisse du système social », dans P. Birnbaum et F. Cazel (dir.), Théorie sociologique, Paris, Presses universitaires de France, 598 p.

Piaget, J. (1968), Le structuralisme, 10 édition, 1992, Paris, Presses universitaires de France, Collection «Que sais-je ?», no 1311.

PIAGET, J. (1978), «La notion de structure», dans UNESCO, La pensée scientifique. Quelques concepts, démarches et méthodes, Paris, Presses de l'Unesco, p. 37-58. 
SAPORTA, B. (1997), «Stratégies des petites et moyennes entreprises», dans Y. Simon et P. Joffre (dir.), Encyclopédie de gestion, tome 3, Paris, Economica, p. 3105-3128.

SCOTT, W.R. (1965), «Fields methods in the study of organizations », dans J.G. March (dir.), Handbook of Organizations, $4^{\mathrm{e}}$ édition, 1972, Chicago, McNally Cy, p. 261304.

SimON, H. (1998), Les champions cachés de la performance, Paris, Dunod.

TAdDÉI, D. et B. CoRIAT (199), Made in France, Paris, Librairie Générale Française, Collection «Livre de Poche $4169 »$, p. 206-215.

TORRÈs, O. (1997), «Pour une approche contingente de la spécificité de la PME », Revue internationale PME, vol. 10, $\mathrm{n}^{\circ}$ 2, p. 9-43.

VAN DE VEN, A. (1989), « Nothing so practical as a good theory », Academy of Management Review, vol. 14, no 4, octobre, p. 486-489.

VÉRAN, L. (1992), «Diagnostics et représentations de l'organisation», dans R. Le Duff et J. Allouche (dir.), Annales du management, Paris, Economica, p. 793-807.

VÉRAN, L. (1995), «Spécificité et outils du diagnostic organisationnel », Économies et sociétés, $\mathrm{SG} \mathrm{n}^{\circ} 21, \mathrm{p} .251-270$.

WEICK, K.E. (1995), « What theory is not, theorizing is », Administrative Science Quarterly, vol. 40, p. 385-390. 